\title{
Solitons in an isolated helix chain
}

\section{Christiansen, Peter Leth; Zolotaryuk, Alexander; Savin, A.V.}

\section{Published in:}

Physical Review E. Statistical, Nonlinear, and Soft Matter Physics

Link to article, DOI:

10.1103/PhysRevE.56.877

Publication date:

1997

\section{Document Version}

Publisher's PDF, also known as Version of record

Link back to DTU Orbit

Citation (APA):

Christiansen, P. L., Zolotaryuk, A., \& Savin, A. V. (1997). Solitons in an isolated helix chain. Physical Review E. Statistical, Nonlinear, and Soft Matter Physics, 56(1), 877-889. https://doi.org/10.1103/PhysRevE.56.877

\section{General rights}

Copyright and moral rights for the publications made accessible in the public portal are retained by the authors and/or other copyright owners and it is a condition of accessing publications that users recognise and abide by the legal requirements associated with these rights.

- Users may download and print one copy of any publication from the public portal for the purpose of private study or research.

- You may not further distribute the material or use it for any profit-making activity or commercial gain

- You may freely distribute the URL identifying the publication in the public portal

If you believe that this document breaches copyright please contact us providing details, and we will remove access to the work immediately and investigate your claim 


\title{
Solitons in an isolated helix chain
}

\author{
P. L. Christiansen, A. V. Zolotaryuk, ${ }^{*}$ and A. V. Savin, ${ }^{\dagger}$ \\ Department of Mathematical Modelling, Technical University of Denmark, DK-2800 Lyngby, Denmark
}

(Received 16 December 1996)

\begin{abstract}
A molecular chain with helix structure has been investigated in the three-dimensional space in the case when it is considered as an isolated object (not subjected to any substrate potential). Each of the chain molecules is allowed to move in three dimensions, and intermolecular interactions (bonds) are assumed to be of the pair type and to have spherical symmetry. The helix structure is provided by the first- and second-neighbor intermolecular bonds as well as by the nearest-neighbor interactions along the longitudinal direction of the chain, stabilizing the helix backbone which can be considered as a generalization of the well-known one-dimensional Fermi-Pasta-Ulam model to include transverse degrees of freedom of the chain molecules. In the particular case of the $\alpha$-helix molecular chain, the intermolecular interactions involved into the model are the point-point bonds connecting the first-, second-, and third-nearest neighbors. The set of nonlinear field equations with respect to the longitudinal and transverse (torsional and radial) displacements of the chain molecules has been derived and treated. Stable nontopological soliton solutions which describe supersonic pulses of longitudinal compression propagating together with localized transverse thickening (bulge) and torsional stretching (untwisting) have been found. The stability properties of these (three-component) soliton solutions have been studied by using numerical techniques developed for seeking solitary-wave solutions in complex molecular systems. [S1063-651X(97)04307-9]
\end{abstract}

PACS number(s): 87.15.-v, 63.20.Ry, 63.20.Pw

\section{INTRODUCTION}

One-dimensional (1D) nonlinear (anharmonic) lattices, the studies of which were originated in a series of pioneering works [1-4], are usually considered as the basic models to describe transport properties in biomolecules $[5,6]$. Any intermolecular potential in such a 1D lattice (chain) has a hardening (positive) anharmonicity. This is a conventional type of anharmonicity in nonlinear lattices and its physical meaning is as follows. When neighboring atoms (or molecules) of the chain are displaced from their equilibrium positions, the repulsion force between them becomes stronger than the harmonic approximation of this interaction. In other words, a hardening anharmonic force contributes to this repulsion with the positive sign. As a result of the presence of such a positive anharmonicity, dynamically stable solitary waves can propagate along the chain with supersonic velocities [2-4]. These nonlinear collective excitations are referred to as lattice (or acoustic) solitons. For some particular choices of the intermolecular potential, like Toda, Boussinesq, etc., the solitons are named accordingly.

In applications to real biological quasi-one-dimensional objects [7-9], the standard 1D Fermi-Pasta-Ulam (FPU) model [1] should be generalized in order to include transverse motions of chain molecules. However, as shown by numerical simulations $[10,11]$, solitonic excitations in anharmonic chains are extremely sensitive to their transverse perturbations and therefore the problem of soliton propagation in 3D objects is far from fully understood. Consequently, the

\footnotetext{
*Also at the Bogolyubov Institute for Theoretical Physics, 252143 Kyiv, Ukraine.

${ }^{\dagger}$ Also at the Institute for Problems of Physics and Technology, 119034 Moscow, Russia.
}

question on the existence and stability of moving solitary waves along realistic biomolecules, considered as 3D objects, is of great interest. In particular, investigations of anharmonic chains, atoms, or molecules which have transverse degrees of freedom $[10,12-17]$ should be mentioned. On the other hand, in some cases transverse displacements of molecules are considered the most important motions in biophysical processes. Thus, in the DNA molecule, the stretching of base pairs in the transverse direction determines the fundamental mechanism of the denaturation of this molecule. The Peyrard-Bishop model of DNA melting [18-20] has just been formulated in terms of only transverse degrees of two complementary strands. Although the DNA molecule is considered in this approach as an isolated object, the model actually describes the 1D dynamics of chain molecules in an effective substrate potential.

The present paper aims to find pure solitary-wave solutions for a helix backbone, the molecules of which are allowed to move in 3D space. All intermolecular interactions are assumed to be of the point-point type. The backbone is considered as an isolated object which is not subjected to any substrate potential. Since only point-point intermolecular interactions are involved, the helix backbone will have a single stabilized ground state in the case if, besides the nearest-neighbor coupling, at least two other bonds are taken into account. For instance, in this paper we consider interactions between the first, second, and nearest neighbors in the longitudinal directions. Particularly, for the $\alpha$-helix macromolecule, these interactions are between the first, second, and third neighbors. In any case of a 3D helix backbone, three intermolecular interactions is the minimal number of bonds required to have a regular structure of the backbone when it is found in an undistorted (ground) state. As shown in Ref. [21], in the 2D case, when the helix backbone is reduced to a planar zigzag chain, two intermolecular interac- 
tions (between the first and second neighbors) are a sufficient number of bonds to create a stable zigzag structure. Note that in the Olsen-Lomdahl-Kerr (OLK) model for the $\alpha$ helix, the two intermolecular interactions (between the first neighbors and the nearest molecules in the longitudinal direction) is not a sufficient number to fix a stable ground state. Any pair of geometric parameters describing the helix structure can be given in terms of the backbone radius, and therefore the helix geometry is arbitrarily scaled by this parameter. Therefore, our generalized version of the OLK model may be considered as the most simple theoretical model of an isolated molecular chain corresponding to realistic situations in biomolecular sciences.

Finally, it should be emphasized that even in the case where molecules are assumed to be coupled by harmonic forces, an effective anharmonicity appears because of the geometry of the system. For breatherlike solutions, the effects of such a geometric nonlinearity have previously been investigated by Cadet [22].

The rest of the paper is organized as follows. In Sec. II we describe a helix model with first- and second-neighbor interactions, including the nearest-neighbor interactions (soft hydrogen bonds) along the longitudinal direction of the backbone. In this section, we describe the geometry of the helix structure, and derive the basic set of three-component equations of motion. The reduction of these equations to lower dimensions is briefly discussed there. In Sec. III, we study the small-amplitude linear limit of the equations of motion. In Sec. IV, we generalize a numerical method developed previously for low dimensions to seek pure solitary-wave solutions of stationary profile in our 3D model. These solutions are chosen in Sec. V as initial conditions for simulations of the equations of motion. The comparison of the initial and final soliton profiles has been carried out there. The concluding remarks on the results of the present paper are outlined in Sec. VI.

\section{A HELIX MODEL}

Let molecules (e.g., amino acids) be linked together in a molecular (polypeptide) chain, as illustrated in Fig. 1, by the first-, second-, and $\nu$-neighbor forces. The forces between the $n$th and $(n+\nu)$ th molecules form a soft (hydrogen) bond in the longitudinal direction of the helix backbone, and therefore the integer $\nu$ (the number of spines) is determined from the condition that the $\nu$-neighboring bond has to be the shortest distance in this direction. This chain has a 3D helical structure, and its molecules are allowed to move in all three $(X, Y, Z)$ directions.

\section{A. Sets of geometric parameters for the description of helix structure}

The geometry of a regular helix backbone, when its molecules are found in equilibrium positions, can be uniquely given by a set of three parameters. To this end, it is convenient to define the positions of the vertices of the helix backbone, using the cylindrical system of coordinates. In this frame the helix structure can be described by (i) the radius $R_{0}$ of the cylinder which spans the helix backbone, (ii) the constant angle $\phi$ in the $X Y$ plane which is formed by each three successive chain molecules (this angle is the projection

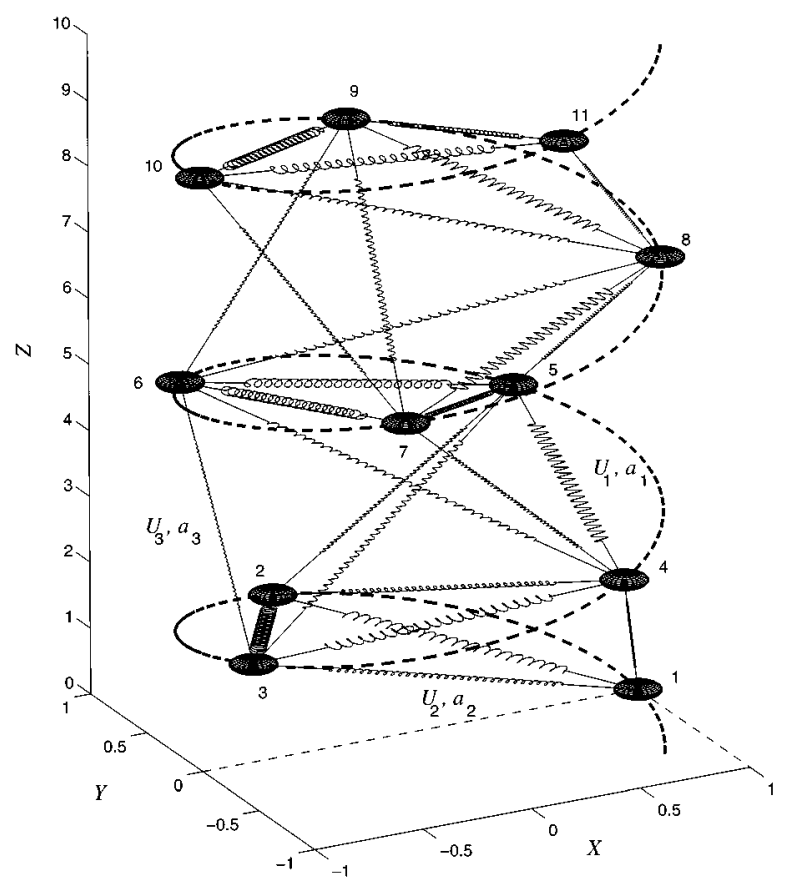

FIG. 1. A fragment of the helix backbone consisting of 11 molecules. The geometry of the backbone is in accordance with the structure of $\alpha$-helix, i.e., $\nu=3$ and $\phi=100^{\circ}$. The intermolecular interactions $U_{j}, j=1,2,3$, are schematically shown by the springs of different diameters. The thicker springs correspond to stronger stiffness of the bonds.

of the valence angle $\psi$ between the nearest valence bonds onto the $X Y$ plane), and (iii) the height $\Delta z$ which measures the $Z$ projection of the distance between the nearestneighboring molecules in the chain. Then the radius vector of each molecule of the helix backbone is given by

$$
\mathbf{R}_{n}=R_{0}(\cos (n \phi), \sin (n \phi), n h), \quad n=0, \pm 1, \ldots,
$$

where $h=\Delta z / R_{0}$. When $\phi>2 \pi / \nu(\phi<2 \pi / \nu)$, we refer to such a chain as a right (left) helix.

Alternatively, the helix can be described by three other parameters, namely, by the distances between (i) the nearest molecules $\left(D_{1}\right)$, (ii) the second neighbors $\left(D_{2}\right)$, and (iii) the $n$th and $(n+\nu)$ th molecules $\left(D_{\nu}\right)$. The length of the vector

$$
\begin{aligned}
& \mathbf{a}_{j n}=\left(\mathbf{R}_{n+j}-\mathbf{R}_{n}\right) / R_{0}=(\cos [(n+j) \phi]-\cos (n \phi), \\
& \qquad \sin [(n+j) \phi]-\sin (n \phi), \\
& \qquad j h), \\
& \text { which connects the } n \text {th and }(n+j) \text { th vertices of the regular } \\
& \text { helix backbone, does not depend on the number of the chain } \\
& \text { site: } \\
& \qquad\left|\mathbf{a}_{j n}\right|=\sqrt{2[1-\cos (j \phi)]+j^{2} h^{2}} \equiv a_{j}=D_{j} / R_{0}, \quad j=1,2, \nu .
\end{aligned}
$$

Using the expression for the distance between the $n$th and $(n+j)$ th vertices of the backbone (3), we find the equation for the angle $\phi$ : 


$$
\frac{3-4 \cos \phi+\cos (2 \phi)}{\nu^{2}-1-\nu^{2} \cos \phi+\cos (\nu \phi)}=\frac{4 a_{1}^{2}-a_{2}^{2}}{\nu^{2} a_{1}^{2}-a_{\nu}^{2}} .
$$

When Eq. (4) has been solved, we obtain

$$
R_{0}=\frac{\sqrt{4 D_{1}^{2}-D_{2}^{2}}}{4 \sin ^{2}(\phi / 2)}, \quad \Delta z=\frac{\sqrt{D_{2}^{2} / 4-D_{1}^{2} \cos ^{2}(\phi / 2)}}{\sin (\phi / 2)} .
$$

Thus there is a one-to-one correspondence between the two sets of parameters: $\left\{R_{0}, \phi, h\right\}$ and $\left\{D_{1}, D_{2}, D_{\nu}\right\}$.

The third set of parameters which may also be adopted for the description of the helix geometry is $\left\{D_{1}, \psi, D_{\nu}\right\}$, where $\psi$ is the valence angle between the nearest-neighboring bonds. The relation between the parameter sets $\left\{R_{0}, \phi, h\right\}$ and $\left\{D_{1}, \psi, D_{\nu}\right\}$ is determined as follows. First, we find the equation for the angle $\phi$ :

$$
\frac{1-\cos (\nu \phi)}{1-\cos \phi}+\left(\nu^{2}-\frac{a_{\nu}^{2}}{a_{1}^{2}}\right) \frac{1-\cos \phi}{1+\cos \psi}=\nu^{2} .
$$

Then the remainder two parameters $R_{0}$ and $h$ are given by

$$
R_{0}=\frac{D_{1} \cos (\psi / 2)}{1-\cos \phi}, \quad \Delta z=D_{1}\left(\frac{|\cos \phi+\cos \psi|}{1-\cos \phi}\right)^{1 / 2} .
$$

Note that the inequality

$$
\cos \phi+\cos \psi<0
$$

is always valid if the integer $\nu>1$.

Since the regular helix structure is given uniquely by three geometric parameters, we need to consider three types of intermolecular interactions which stabilize this structure. One of these has to be introduced, as usual, between the nearest neighbors along the helix chain and it may be referred to as valence bonds. The second type of interactions couples the molecules situated along the longitudinal direction of the chain as in the OLK model [10]. It is responsible for the secondary structure of the chain macromolecule. Thus in protein these interactions are called hydrogen bonds. However, as shown above, in order to have a stable helix backbone when the intermolecular interactions are spherically symmetric (i.e., if only point-point central interactions are assumed), besides the interactions between the nearest neighbors along the helix backbone and along the longitudinal direction, we have to consider some third type of intermolecular bonds. For instance, it can be the three-particle interaction fixing a certain valence angle $\psi$. The simplest and most straightforward way to take this fact into account is to consider the interaction between the $n$th and $(n+2)$ th molecules, i.e., the second-neighbor coupling. In this respect, such a helix will be the most simple generalization of the FPU chain considered as a 3D object.

Now we need to discuss the number of spines $\nu$. This integer should be chosen in such a way that the length of longitudinal (hydrogen) bonds would be the shortest distance between the $n$th and $(n+\nu)$ th molecules. Using the first equation in Eq. (7), we exclude the variable $a_{1}$ in Eq. (6), and find the relation

$$
\frac{a_{\nu}^{2}}{2}=1-\cos (\nu \phi)-\nu^{2} \frac{(\cos \phi+\cos \psi)(1-\cos \phi)}{1+\cos \psi},
$$

which gives the dependence of the distance $a_{\nu}$ on the integer $\nu$. The minimization of the distance $a_{\nu}=a_{\nu}(\phi, \psi)$ with respect to all integers $\nu \geqslant 3$ at given values of the angles $\phi$ and $\psi$ yields the required integer $\nu$. Thus, for the $\alpha$-helix macromolecule we have $\phi=100^{\circ}$ and $\psi=110^{\circ}$. In this case, the minimal distance $a_{\nu}$ occurs at $\nu=3$.

In the case of three spines (e.g., in protein), when $\nu=3$, it is interesting to consider the equidistance case when $D_{1}=D_{2}=D_{3}$. In this particular case Eqs. (3) and (4) can be solved explicitly. As a result, we find the angle $\phi: \sin (\phi / 2)$ $=\sqrt{5 / 6}$. The other two parameters are given by $a_{1}=a_{2}$ $=a_{3}=10 / 3 \sqrt{3}$ and $h=\frac{1}{3} \sqrt{10 / 3}$. Since the angle $\phi$ exceeds the value $2 \pi / 3$, this particular case corresponds to a right helix.

\section{B. Equations of motion}

The total Hamiltonian of the described helix backbone with three types of interactions, which link the first and second neighbors as well as the nearest neighbors along the longitudinal direction of the chain, can be written in the form

$$
H=\sum_{n}\left[\frac{1}{2} M\left(\dot{x}_{n}^{2}+\dot{y}_{n}^{2}+\dot{z}_{n}^{2}\right)+K R_{0}^{2} \sum_{j=1,2, \nu} U_{j}\left(r_{j n}\right)\right]
$$

where $M$ is the mass of chain molecules, $K$ is the characteristic stiffness of the intermolecular forces, the coordinates $x_{n}, y_{n}$, and $z_{n}$ describe the displacements of the $n$th molecule from its equilibrium position given in the $X Y Z$ frame by the radius vector (1), and the dots denote the differentiation with respect to time $t$. Each of the three functions $U_{j}\left(r_{j n}\right)$ 's, which are assumed, in general, to be different, describes an intermolecular interaction between the nearestneighboring $(j=1)$, second-neighboring $(j=2)$, and $(\nu)$ neighboring $(j=\nu)$ molecules. These interactions are assumed to depend only on the dimensionless intermolecular distances $r_{j n}=R_{j n} / R_{0}$ where $R_{j n}$ is the distance between the $n$th and $(n+j)$ th molecules. The intermolecular potentials $U_{j}(r), j=1,2, \nu$, are normalized by $U_{j}\left(a_{j}\right)=0, U_{j}^{\prime}\left(a_{j}\right)=0$, and they have the standard form, like the Lennard-Jones interaction. In order to deal with both the harmonic approximation and with more realistic potentials, and to have a parameter of intermolecular nonlinearity (anharmonicity), we will use the Morse potentials

$$
\begin{aligned}
U_{j}(r) & =\frac{1}{2}\left(\kappa_{j} / \gamma_{j}^{2}\right)\left\{1-\exp \left[-\gamma_{j}\left(r-a_{j}\right)\right]\right\}^{2} \\
& =\frac{1}{2} \kappa_{j}\left(r-a_{j}\right)^{2}\left[1-\gamma_{j}\left(r-a_{j}\right)+\cdots\right], \quad j=1,2, \nu,
\end{aligned}
$$

where $\kappa_{j}=K_{j} / K=U_{j}^{\prime \prime}\left(a_{j}\right)$ is the dimensionless stiffness constant of the bond connecting the $n$th and $(n+j)$ th molecules, while $\gamma_{j}$ is the anharmonicity parameter of this bond. In the limit $\gamma_{j} \rightarrow 0$, potentials (11) describe the harmonic approximation.

For the dimensionless description it is convenient to introduce the normalized time

$$
\tau=\omega_{0} t, \quad \omega_{0}=\sqrt{K / M},
$$




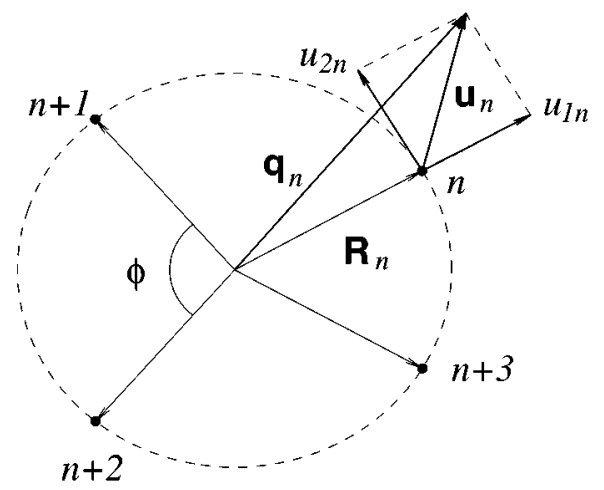

FIG. 2. The frame of local normal and tangent coordinates in the $X Y$ plane.

and the dimensionless vectors $\mathbf{q}_{n}$ 's defined by

$$
\begin{gathered}
\mathbf{q}_{n}\left(q_{1 n}, q_{2 n}, q_{3 n}\right)=\mathbf{R}_{n} / R_{0}+\mathbf{v}_{n}, \\
\mathbf{v}_{n}=\left(v_{1 n}, v_{2 n}, v_{3 n}\right)=\left(x_{n}, y_{n}, z_{n}\right) / R_{0} .
\end{gathered}
$$

Then the distances $r_{j n}$ are represented as

$$
r_{j n}=\left|\mathbf{q}_{n+j}-\mathbf{q}_{n}\right|
$$

and the Hamiltonian (10) can be rewritten in the dimensionless form. Given in the units of $K R_{0}^{2}$, it takes the form

$$
\mathcal{H}=\sum_{n}\left[\frac{1}{2}\left(\frac{d \mathbf{q}_{n}}{d \tau}\right)^{2}+\sum_{j} U_{j}\left(\left|\mathbf{q}_{n+j}-\mathbf{q}_{n}\right|\right)\right] .
$$

The equations of motion which correspond to the Hamiltonian (15) take the following form:

$$
\frac{d^{2} \mathbf{q}_{n}}{d \tau^{2}}=\sum_{j}\left[W_{j}\left(r_{j n}\right)\left(\mathbf{q}_{n+j}-\mathbf{q}_{n}\right)-W_{j}\left(r_{j, n-j}\right)\left(\mathbf{q}_{n}-\mathbf{q}_{n-j}\right)\right],
$$

where the functions $W_{j}\left(r_{j n}\right)$ are defined by

$$
W_{j}\left(r_{j n}\right)=\frac{U_{j}^{\prime}\left(r_{j n}\right)}{r_{j n}}
$$

In Eq. (16) and in the following, the summation over $j$ $=1,2, \nu$ is not indicated explicitly, except for the particular cases when the number of spines $\nu$ is specified.

\section{Local frame of coordinates}

It is more natural to describe the displacements of molecules from the equilibrium positions locally at each molecule. More precisely, at each equilibrium position of the $n$th molecule, we consider the normal and tangent (to the circle in the $X Y$ plane, as shown in Fig. 2) components of the displacement vector $\mathbf{v}_{n}$ [see Eqs. (13)]. We denote this vector in the local system by $\mathbf{u}_{n}=\left\{u_{1 n}, u_{2 n}, u_{3 n}\right\}$, where $u_{1 n}$ and $u_{2 n}$ are the normal and tangent projections of the vector $\mathbf{u}_{n}$, respectively. The longitudinal coordinate is not transformed, so that $u_{3 n} \equiv v_{3 n}$. Therefore, both the systems of coordinates are related by the rotational transformation $\mathbf{T}_{n}$ defined by

$$
\mathbf{T}_{n} \mathbf{v}_{n}=\mathbf{u}_{n}, \quad \mathbf{T}_{n}=\left(\begin{array}{ccc}
\cos (n \phi) & \sin (n \phi) & 0 \\
-\sin (n \phi) & \cos (n \phi) & 0 \\
0 & 0 & 1
\end{array}\right)
$$

The set of the unitary operators $\mathbf{T}_{n}$ 's forms a group: $\mathbf{T}_{m} \mathbf{T}_{n}$ $=\mathbf{T}_{m+n}$, with the unity $\mathbf{T}_{0}=\mathbf{I}$, where $\mathbf{I}$ is the unity operator.

The substitution of the expression $\mathbf{q}_{n}=\mathbf{R}_{n} / R_{0}+\mathbf{T}_{n}^{-1} \mathbf{u}_{n}$ [see Eqs. (13) and (18)] into the equations of motion (16) yields

$$
\frac{d^{2} \mathbf{u}_{n}}{d \tau^{2}}=\sum_{j}\left[\mathbf{T}_{j}^{-1} \mathbf{F}_{j}\left(\mathbf{u}_{n}, \mathbf{u}_{n+j}\right)-\mathbf{F}_{j}\left(\mathbf{u}_{n-j}, \mathbf{u}_{n}\right)\right],
$$

where the intermolecular forces $\mathbf{F}_{j}$ 's are defined by

$$
\mathbf{F}_{j}\left(\mathbf{u}_{n}, \mathbf{u}_{n+j}\right)=W_{j}\left(r_{j n}\right)\left(\mathbf{c}_{j}+\mathbf{u}_{n+j}-\mathbf{T}_{j} \mathbf{u}_{n}\right) .
$$

Here the distance $r_{j n}$ between the $n$th and $(n+j)$ th molecules is represented by

$$
r_{j n}=\left|\mathbf{a}_{j n}+\mathbf{T}_{n+j}^{-1} \mathbf{u}_{n+j}-\mathbf{T}_{n}^{-1} \mathbf{u}_{n}\right|,
$$

and the constant vectors $\mathbf{c}_{j}$ 's are defined by

$$
\mathbf{c}_{j}=(1-\cos (j \phi), \sin (j \phi), j h) \text {. }
$$

As can be seen from Eqs. (20) and (21), the forces $\mathbf{F}_{j}$ 's are not expressed in terms of the differences of the vectors $\mathbf{u}_{n}$ and $\mathbf{u}_{n+j}$ because of the presence of the operators $\mathbf{T}_{j}$ 's in these expressions.

\section{Lower dimensions}

Consider some particular cases corresponding to lower (one and two) dimensions, e.g., taking $\nu=1$ or $\nu=2$. First, we note that the equations of motion for the $1 \mathrm{D}$ case can be obtained from Eqs. (2) and (17)-(22) if we put there $\phi$ $=2 \pi$. Particularly, we obtain $\mathbf{a}_{j n}=(0,0, j h)$ and $\mathbf{T}_{n}=\mathbf{I}$. Reducing to the $1 \mathrm{D}$ case gives $\mathbf{v}_{n}=\mathbf{u}_{n}=\left(0,0, u_{n}\right)$ and $\mathbf{q}_{n+j}$ $-\mathbf{q}_{n}=\left(0,0, r_{j n}\right)$, where $r_{j n}=j h+u_{n+j}-u_{n}$. As a result, Eq. (19) is reduced to

$$
\frac{d^{2} u_{n}}{d \tau^{2}}=\sum_{j}\left[U_{j}^{\prime}\left(j h+u_{n+j}-u_{n}\right)-U_{j}^{\prime}\left(j h+u_{n}-u_{n-j}\right)\right] .
$$

In the simplest case $\nu=1$, this equation describes the standard FPU model, while for $j>1$ we obtain the 1D generalization of this model including long-range intermolecular interactions. For the particular case of the first- and secondneighbor interactions, $\nu=2(j=1,2)$, this model has been extensively studied in Refs. [23,24].

Similarly, putting $\phi=\pi$, we reduce the 3D helix backbone to a planar zigzag chain studied previously in Ref. [21]. Indeed, in the case with the first- and second-neighbor interactions $(\nu=2)$, we obtain

$$
\begin{gathered}
\mathbf{a}_{1 n}=\left(2(-1)^{n+1}, 0, h\right) \quad \mathbf{a}_{2 n}=(0,0,2 h), \\
\mathbf{T}_{n}=\left(\begin{array}{ccc}
(-1)^{n} & 0 & 0 \\
0 & (-1)^{n} & 0 \\
0 & 0 & 1
\end{array}\right) .
\end{gathered}
$$


Let $\mathbf{u}_{n}=\left(u_{n}, 0, \beta_{n}\right)$, with $u_{n}(\tau)$ and $\beta_{n}(\tau)$ being generalized coordinates. Then in terms of these coordinates, the first- and second-neighbor distances are

$$
\begin{gathered}
r_{1 n}=\sqrt{\left(2+u_{n}+u_{n+1}\right)^{2}+\left(h+\beta_{n+1}-\beta_{n}\right)^{2}}, \\
r_{2 n}=\sqrt{\left(u_{n+2}-u_{n}\right)^{2}+\left(2 h+\beta_{n+2}-\beta_{n}\right)^{2}},
\end{gathered}
$$

respectively. The corresponding equations of motion for the lattice fields $u_{n}(\tau)$ and $\beta_{n}(\tau)$ are derived immediately from the Lagrangian

$$
\mathcal{L}=\sum_{n}\left[\frac{1}{2}\left(\frac{d u_{n}}{d \tau}\right)^{2}+\frac{1}{2}\left(\frac{d \beta_{n}}{d \tau}\right)^{2}-\sum_{j=1,2} U_{j}\left(r_{j n}\right)\right] .
$$

As was shown in Ref. [21], these equations admit stable two-component nontopological soliton solutions.

\section{SMALL-AMPLITUDE WAVES}

In the harmonic approximation for all of the intermolecular forces $\left(\gamma_{j} \rightarrow 0, j=1,2, \nu\right)$, we obtain the linear expansion

$$
\mathbf{F}_{j}\left(\mathbf{u}_{n}, \mathbf{u}_{n+j}\right)=\alpha_{j}\left\langle\mathbf{u}_{n+j}-\mathbf{T}_{j} \mathbf{u}_{n}, \mathbf{c}_{j}\right\rangle \mathbf{c}_{j}+\cdots,
$$

where $\alpha_{j}=\kappa_{j} / a_{j}^{2}$ and \langle\rangle denotes the inner product. Consequently, the linearized equations of motion take the form

$$
\begin{aligned}
\frac{d^{2} \mathbf{u}_{n}}{d \tau^{2}}= & \sum_{j} \alpha_{j}\left[\left\langle\mathbf{u}_{n+j}-\mathbf{T}_{j} \mathbf{u}_{n}, \mathbf{c}_{j}\right\rangle \mathbf{T}_{j}^{-1} \mathbf{c}_{j}\right. \\
& \left.-\left\langle\mathbf{u}_{n}-\mathbf{T}_{j} \mathbf{u}_{n-j}, \mathbf{c}_{j}\right\rangle \mathbf{c}_{j}\right] .
\end{aligned}
$$

Substituting the plane wave

$$
\mathbf{u}_{n}=\mathbf{A}_{n} \exp [i(k n-\Omega \tau)]
$$

into Eqs. (28), we obtain the following dispersion law:

$$
\left|\begin{array}{ccc}
\Omega^{2}-c_{11} & -i c_{12} & -i c_{13} \\
i c_{12} & \Omega^{2}-c_{22} & -c_{23} \\
i c_{13} & -c_{23} & \Omega^{2}-c_{33}
\end{array}\right|=0,
$$

with the coefficients

$$
\begin{gathered}
c_{11}=2 \sum_{j} \alpha_{j}[1-\cos (j \phi)]^{2}[1+\cos (j k)], \\
c_{12}=2 \sum_{j} \alpha_{j}[1-\cos (j \phi)] \sin (j \phi) \sin (j k), \\
c_{13}=2 \sum_{j} \alpha_{j}(j h)[1-\cos (j \phi)] \sin (j k), \\
c_{22}=2 \sum_{j} \alpha_{j} \sin ^{2}(j \phi)[1-\cos (j k)], \\
c_{23}=2 \sum_{j} \alpha_{j}(j h) \sin (j \phi)[1-\cos (j k)], \\
c_{33}=2 \sum_{j} \alpha_{j}(j h)^{2}[1-\cos (j k)] .
\end{gathered}
$$

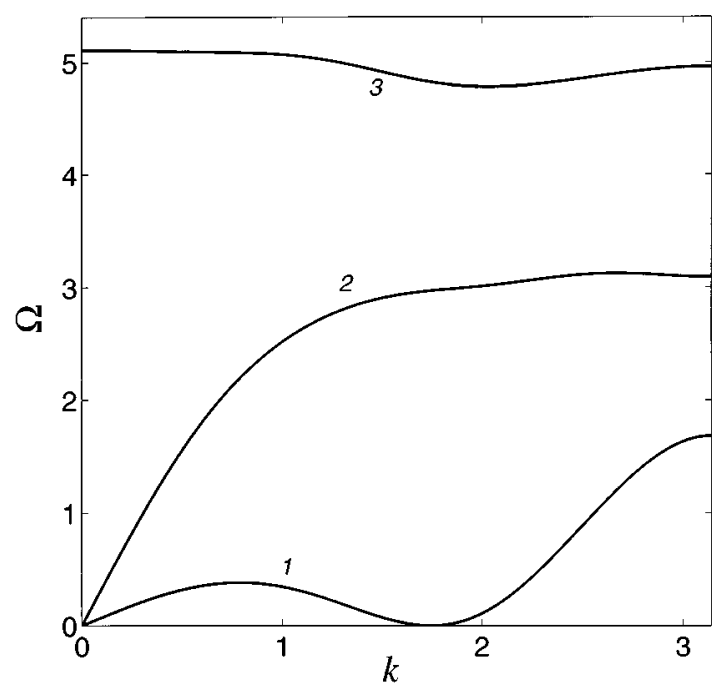

FIG. 3. The frequencies $\Omega_{t}$ (curve 1), $\Omega_{l}$ (curve 2), and $\Omega_{\text {op }}$ (curve 3) against wave number $k, 0 \leqslant k \leqslant \pi$, for the chain with the parameter set (35).

Explicitly, Eq. (30) can be rewritten as

$$
\begin{aligned}
& \Omega^{6}-\left(c_{11}+c_{22}+c_{33}\right) \Omega^{4}+\left(c_{11} c_{22}+c_{11} c_{33}+c_{22} c_{33}-c_{12}^{2}-c_{13}^{2}\right. \\
& \left.-c_{23}^{2}\right) \Omega^{2}+\left(c_{11} c_{23}^{2}+c_{22} c_{13}^{2}+c_{33} c_{12}^{2}-c_{11} c_{22} c_{33}\right. \\
& \left.\quad-2 c_{12} c_{13} c_{23}\right)=0 .
\end{aligned}
$$

Using the explicit form of Eqs. (30)-(32), one can be convinced of the existence of three nondegenerate and nonnegative roots of the cubic (with respect to $\Omega^{2}$ ) equation (32) for all $0<k \leqslant \pi$. In the long-wavelength limit $k \rightarrow 0$, the free term, and the coefficient at $\Omega^{2}$ in the dispersion equation (32) tend to zero. Therefore, two of three solutions of this equation are acoustic branches. These two $\Omega_{l}(k)$ and $\Omega_{t}(k)$ (see Fig. 3) correspond to the longitudinal and torsional oscillations of the chain molecules, respectively. The third root of Eq. (32) gives an optical branch $\Omega_{\text {op }}(k)$, corresponding to the transverse oscillations of molecules in the radial direction of the helix backbone. Explicitly, at $k=0$ we have

$$
\Omega_{\mathrm{op}}^{2}(0)=c_{11}+c_{22}+c_{33}=4 \sum_{j} \alpha_{j}[1-\cos (j \phi)]^{2} .
$$

The presence of two acoustic branches should result in the existence of two speeds of sound: longitudinal $\left(v_{l}\right)$ and torsional $\left(v_{t}\right)$. In dimensionless form, they can be defined as the limits

$$
s_{l, t}=\frac{v_{l, t}}{v_{0}}=h \lim _{k \rightarrow 0} \frac{\Omega_{l, t}(k)}{k},
$$

where $v_{0}=(K / M)^{1 / 2} R_{0}$ is the characteristic velocity of small-amplitude waves in the helix backbone.

For numerical computations we choose the following values of the system parameters:

$$
\nu=3, \quad \phi=100^{\circ}, \quad h=1, \quad \kappa_{1}=10, \quad \kappa_{2}=5, \quad \kappa_{3}=1 .
$$


In the parameter set (35), the values for the number $\nu$ and the angle $\phi$ correspond to the $\alpha$-helix protein molecule. The stiffness constants $\kappa_{1}, \kappa_{2}$, and $\kappa_{3}$ are related each to other approximately as the elasticities of the valence bond, valence angle, and hydrogen bond. The form of all of the three dispersion curves for the values (35) is shown in Fig. 3. At $k$ $=0$, the frequencies are $\Omega_{l}=\Omega_{t}=0$ and $\Omega_{\mathrm{op}}=5.11$. It follows from the explicit representation of the coefficients (31) that at a certain value of the wave number $k=k_{0}$, the free term in Eq. (32) becomes zero. This means that, at this value, softening the torsional oscillations happens $\left[\Omega_{t}\left(k_{0}\right)=0\right]$. For the parameter set (35) this value is $k_{0}=1.748$. As follows from Fig. 3, the frequency spectrum of the helix chain consists of one separate optical zone and two acoustic zones. The frequency spectrum of the torsional oscillations lies inside the frequency spectrum of the longitudinal oscillations. Moreover, the velocity of the longitudinal sound $s_{l}=3.39$ significantly exceeds the speed of the torsional sound $s_{t}$ $=0.75$.

\section{A SOLITON ANALYSIS}

In this section we develop a numerical scheme for seeking solitary-wave solutions of the stationary profile for the basic equations of motion (16). This scheme can be applied if the profile of such solutions appears to be sufficiently smooth, though varied from site to site along the chain. In order to study discreteness effects, we should use more complicated numerical techniques such as the pseudospectral method suggested by Eilbeck and Flesch [25], later developed by Duncan et al. [26]. When the soliton solutions have been found, then they can be chosen as initial conditions for numerical simulations of these equations. The final profile of the vector lattice field $\mathbf{q}_{n}(\tau)$ obtained under the simulations at sufficiently large times $\tau$ allows us to conclude whether or not the initial soliton profile is a stable solution of Eqs. (16). The main point in such a numerical approach is an appropriate choice of a discrete functional in the numerical scheme. As was shown previously [21], such a functional can be constructed from the corresponding Lagrangian of the system.

To accomplish the soliton analysis of the equations of motion (16), we treat them in the cylindrical system of coordinates. Therefore, we write

$$
\begin{gathered}
q_{1 n}=\left(1+\eta_{n}\right) \cos \left(n \phi+\theta_{n}\right), \\
q_{2 n}=\left(1+\eta_{n}\right) \sin \left(n \phi+\theta_{n}\right), \\
q_{3 n}=n h+\beta_{n}
\end{gathered}
$$

where $\eta_{n}$ describes the radial displacement of the $n$th molecule from the cylinder surface which spans the helix backbone when its molecules are situated at the equilibrium positions. It is positive if the displacement is outside, and the thickening of the helix occurs in this place. If the displacement is directed inside the helix, then $\eta_{n}$ is negative. The second generalized coordinate $\theta_{n}$ describes the azimuthal deviation of the $n$th molecule from its equilibrium position, and $\beta_{n}$ is the $Z$ coordinate of the displacement. Then the Lagrangian of the helix backbone written in terms of these new variables takes the form

$$
\begin{aligned}
\mathcal{L}= & \mathcal{L}\left\{\frac{d \eta_{n}}{d \tau}, \eta_{n} ; \frac{d \theta_{n}}{d \tau}, \theta_{n} ; \frac{d \beta_{n}}{d \tau}, \beta_{n}\right\}=\sum_{n}\left\{\frac { 1 } { 2 } \left[\left(\frac{d \eta_{n}}{d \tau}\right)^{2}\right.\right. \\
& \left.\left.+\left(1+\eta_{n}\right)^{2}\left(\frac{d \theta_{n}}{d \tau}\right)^{2}+\left(\frac{d \beta_{n}}{d \tau}\right)^{2}\right]-\sum_{j} U_{j}\left(r_{j n}\right)\right\},
\end{aligned}
$$

where the distance $r_{j n}$ is given by

$$
\begin{aligned}
r_{j n}^{2}= & \left(1+\eta_{n}\right)^{2}+\left(1+\eta_{n+j}\right)^{2}-2\left(1+\eta_{n}\right)\left(1+\eta_{n+j}\right) \\
& \times \cos \left(j \phi+\theta_{n+j}-\theta_{n}\right)+\left(j h+\beta_{n+j}-\beta_{n}\right)^{2} .
\end{aligned}
$$

The corresponding equations of motion are

$$
\begin{aligned}
\frac{d^{2} \eta_{n}}{d \tau^{2}}= & \left(1+\eta_{n}\right)\left(\frac{d \theta_{n}}{d \tau}\right)^{2}-\sum_{j}\left\{W _ { j } ( r _ { j , n - j } ) \left[1+\eta_{n}\right.\right. \\
& \left.-\left(1+\eta_{n-j}\right) \cos \left(j \phi+\theta_{n}-\theta_{n-j}\right)\right]+W_{j}\left(r_{j n}\right)\left[1+\eta_{n}\right. \\
& \left.\left.-\left(1+\eta_{n+j}\right) \cos \left(j \phi+\theta_{n+j}-\theta_{n}\right)\right]\right\}, \\
\frac{d^{2} \theta_{n}}{d \tau^{2}}= & \frac{1}{1+\eta_{n}}\left\{-2 \frac{d \eta_{n}}{d \tau} \frac{d \theta_{n}}{d \tau}+\sum_{j}\left[W_{j}\left(r_{j n}\right)\left(1+\eta_{n+j}\right)\right.\right. \\
& \times \sin \left(j \phi+\theta_{n+j}-\theta_{n}\right)-W_{j}\left(r_{j, n-j}\right)\left(1+\eta_{n-j}\right) \\
& \left.\left.\times \sin \left(j \phi+\theta_{n}-\theta_{n-j}\right)\right]\right\}, \\
\frac{d^{2} \beta_{n}}{d \tau^{2}=} & \sum_{j}\left[W_{j}\left(r_{j n}\right)\left(j h+\beta_{n+j}-\beta_{n}\right)-W_{j}\left(r_{j, n-j}\right)\left(j h+\beta_{n}\right.\right. \\
& \left.\left.-\beta_{n-j}\right)\right] .
\end{aligned}
$$

We assume that soliton solutions have moving permanent profile, i.e., we put $\eta_{n}=\eta(n h-s \tau), \theta_{n}=\theta(n h-s \tau)$, and $\beta_{n}=\beta(n h-s \tau)$, where $s=v / v_{0}$ with $v_{0}$ being the characteristic sound velocity defined in Sec. III. As illustrated by Fig. 3, there are three types of waves: one optical and two acoustic modes. Therefore, there is no need to take into account the dispersion of the optical mode, and therefore we can approximate the first and second time derivatives of $\eta_{n}$ by the simplest spatial difference derivatives as follows:

$$
\begin{gathered}
\frac{d \eta_{n}}{d \tau}=-s \eta^{\prime}(n h-s \tau) \simeq-s\left(\eta_{n+1}-\eta_{n-1}\right) / 2 h, \\
\frac{d^{2} \eta_{n}}{d \tau^{2}}=s^{2} \eta^{\prime \prime}(n h-s \tau) \simeq s^{2}\left(\eta_{n+1}-2 \eta_{n}+\eta_{n-1}\right) / h^{2} .
\end{gathered}
$$

However, for the longitudinal and torsional displacements we need to take into account the dispersion which arises from the discreteness of the chain backbone. To this end, we represent the time derivatives of $\theta_{n}$ and $\beta_{n}$ by differences which additionally contain higher-order spatial difference derivatives chosen in such a way that they cancel the higher 
expansion terms while passing to the continuum limit. Thus, introducing the relative displacements $\varphi_{n}=\theta_{n+1}-\theta_{n}$ and $\rho_{n}=\beta_{n+1}-\beta_{n}$, we can write

$$
\begin{aligned}
& \frac{d \theta_{n}}{d \tau}=-s \theta^{\prime}(n h-s \tau) \simeq-s\left(\frac{\theta_{n+1}-\theta_{n-1}}{2 h}\right. \\
&\left.-\frac{\theta_{n+2}-3 \theta_{n+1}+3 \theta_{n}-\theta_{n-1}}{6 h}\right) \\
&= s\left(\theta_{n+2}-6 \theta_{n+1}+3 \theta_{n}+2 \theta_{n-1}\right) / 6 h \\
&= s\left(\varphi_{n+1}-5 \varphi_{n}-2 \varphi_{n-1}\right) / 6 h, \\
& \frac{d^{2} \theta_{n}}{d \tau^{2}=} s^{2} \theta^{\prime \prime}(n h-s \tau) \simeq s^{2}\left(\frac{\theta_{n+1}-2 \theta_{n}+\theta_{n-1}}{h^{2}}\right. \\
&-\left.\frac{\theta_{n+2}-4 \theta_{n+1}+6 \theta_{n}-4 \theta_{n-1}+\theta_{n-2}}{12 h^{2}}\right) \\
&=-s^{2}\left(\varphi_{n+1}-15 \varphi_{n}+15 \varphi_{n-1}-\varphi_{n-2}\right) / 12 h^{2}, \\
&\left.+\rho_{n-2}\right) / 12 h^{2} . \\
& \frac{d^{2} \beta_{n}}{d \tau^{2}}=s^{2} \beta^{\prime \prime}(n h-s \tau) \simeq-s^{2}\left(\rho_{n+1}-15 \rho_{n}+15 \rho_{n-1}\right. \\
&=s\left(\beta_{n}\right.
\end{aligned}
$$

Using the discretized versions (42)-(46), we derive from the dynamical equations (39)-(41) the following discrete equations for the displacements $\eta_{n}, \varphi_{n}$, and $\rho_{n}$ :

$$
\begin{aligned}
\mathcal{F}_{1 n} \equiv & \frac{s^{2}}{h^{2}}\left[\eta_{n+1}-2 \eta_{n}+\eta_{n-1}-\left(1+\eta_{n}\right)\left(\varphi_{n+1}-5 \varphi_{n}\right.\right. \\
& \left.\left.-2 \varphi_{n-1}\right)^{2} / 36\right]+\sum_{j}\left\{W _ { j } ( r _ { j , n - j } ) \left[1+\eta_{n}\right.\right. \\
& \left.-\left(1+\eta_{n-j}\right) \cos \left(j \phi+\sum_{i=1}^{j} \varphi_{n-j+i-1}\right)\right]+W_{j}\left(r_{j n}\right) \\
& \left.\times\left[1+\eta_{n}-\left(1+\eta_{n+j}\right) \cos \left(j \phi+\sum_{i=1}^{j} \varphi_{n+i-1}\right)\right]\right\}=0,
\end{aligned}
$$

$$
\begin{aligned}
\mathcal{F}_{2 n} \equiv & \frac{s^{2}}{12 h^{2}}\left[\left(1+\eta_{n}\right)\left(\varphi_{n+1}-15 \varphi_{n}+15 \varphi_{n-1}-\varphi_{n-2}\right)\right. \\
& \left.+2\left(\eta_{n+1}-\eta_{n-1}\right)\left(\varphi_{n+1}-5 \varphi_{n}-2 \varphi_{n-1}\right)\right] \\
& +\sum_{j}\left[W_{j}\left(r_{j n}\right)\left(1+\eta_{n+j}\right) \sin \left(j \phi+\sum_{i=1}^{j} \varphi_{n+i-1}\right)\right. \\
& \left.-W_{j}\left(r_{j, n-j}\right)\left(1+\eta_{n-j}\right) \sin \left(j \phi+\sum_{i=1}^{j} \varphi_{n-j+i-1}\right)\right]=0 \\
& \frac{s^{2}}{12 h^{2}}\left(\rho_{n+1}-15 \rho_{n}+15 \rho_{n-1}-\rho_{n-2}\right) \\
& +\sum_{j}\left[W_{j}\left(r_{j n}\right)\left(j h+\sum_{i=1}^{j} \rho_{n+i-1}\right)-W_{j}\left(r_{j, n-j}\right)\right. \\
& \left.\times\left(j h+\sum_{i=1}^{j} \rho_{n-j+i-1}\right)\right]=0 .
\end{aligned}
$$

The last equation can be integrated and, as a result, it is transformed to

$$
\begin{gathered}
\mathcal{F}_{3 n} \equiv \frac{s^{2}}{12 h^{2}}\left(\rho_{n+1}-14 \rho_{n}+\rho_{n-1}\right)+\sum_{j} \sum_{l=1}^{j} W_{j}\left(r_{j, n-j+l}\right) \\
\times\left(j h+\sum_{i=1}^{j} \rho_{n-j+l+i-1}\right)=0
\end{gathered}
$$

The system of the discrete equations (47), (48), and (50) was solved numerically in the particular case of the $\alpha$ helix $(\nu=3)$ macromolecule. Our aim was to find only pure soliton solutions of this system. Each of these solutions $\left\{\eta_{n}, \varphi_{n}, \rho_{n}\right\}_{n=1}^{N}$ smoothly depends on the chain site $n$, and has zero asymptotics at the chain ends. It is convenient to seek the first approximation of such a solution as a minimum of the functional

$$
\mathcal{F}=\frac{1}{2} \sum_{n=4}^{N-3}\left(\mathcal{F}_{1 n}^{2}+\mathcal{F}_{2 n}^{2}+\mathcal{F}_{3 n}^{2}\right),
$$

where $N$ is the number of the chain sites. The problem for the conditional minimum

$$
\mathcal{F} \rightarrow \min : \quad \eta_{n}=\varphi_{n}=\rho_{n}=0, \quad n=1,2,3, N-2, N-1, N,
$$

was solved numerically by using the Broyden-FletcherGoldfarb-Shanno variable metric algorithm [27]. The initial point was taken in the form of the bell-shaped pulses

$$
\begin{aligned}
& \eta_{n}=A_{n} / \cosh ^{2}[\mu(n-N / 2)], \\
& \varphi_{n}=A_{\varphi} / \cosh ^{2}[\mu(n-N / 2)],
\end{aligned}
$$




$$
\rho_{n}=A_{\rho} / \cosh ^{2}[\mu(n-N / 2)]
$$

where $\mu$ is an adjustable parameter. It describes the width of the pulse chosen as a starting point for the minimization procedure. The parameters $A_{\eta}, A_{\varphi}$, and $A_{\rho}$ are the amplitudes of this starting pulse. It is necessary to choose the number of sites $N$ approximately ten times larger than the width of the solution. In this case, the shape of the solution will be not affected by the chain ends. We took $N=200$, and this number was appropriate for finding sufficiently broad soliton solutions.

Since the surface $\mathcal{F}=\mathcal{F}\left(\eta_{4}, \ldots, \eta_{N-3}\right.$; $\left.\varphi_{4}, \ldots, \varphi_{N-3} ; \rho_{4}, \ldots, \rho_{N-3}\right)$ is strongly ravined, the search of soliton solutions as a minimum of the function (51) leads to slowly convergent numerical procedure. Therefore the final shape of the soliton solution was found as a numerical solution of the system of $3(N-6)$ nonlinear equations (47), (48), and (50) with respect to the variables $\left\{\eta_{n}, \varphi_{n}, \rho_{n}\right\}_{n=4}^{N-3}$ where $\eta_{n}=\varphi_{n}=\rho_{n}=0$ if $n=1,2,3, N-2, N-1, N$. A modification of the Powell hybrid method including the program HYBRD from the packet of subprograms MINPACK was used for these purposes. Each minimum point obtained under solving the minimization problem (52) was used as an initial point in this method. The necessary condition for the present numerical scheme to be applied for seeking soliton solutions is their smooth dependence on $n$. In the class of such solutions, the method allows us to find their profiles and determine the region of parameter values where soliton solutions exist. The absence of this type of solutions to the set of Eqs. (47), (48), and (50) implies the absence of soliton solutions of the basic system of the equations of motion (16).

Besides the velocity $s$, it is convenient to describe threecomponent soliton solutions $\left\{\eta_{n}, \varphi_{n}, \rho_{n}\right\}_{n=1}^{N}$, obtained numerically by solving the system of Eqs. (47), (48), and (50), by their energy

$$
\begin{aligned}
E= & \sum_{n=4}^{N-3}\left\{\frac { s ^ { 2 } } { 8 h ^ { 2 } } \left[\left(\eta_{n+1}-\eta_{n-1}\right)^{2}+\frac{1}{9}\left(1+\eta_{n}\right)^{2}\left(\varphi_{n+1}-5 \varphi_{n}\right.\right.\right. \\
& \left.\left.\left.-2 \varphi_{n-1}\right)^{2}+\frac{1}{9}\left(\rho_{n+1}-5 \rho_{n}-2 \rho_{n-1}\right)^{2}\right]+\sum_{j=1}^{3} U_{j}\left(r_{j n}\right)\right\}
\end{aligned}
$$

(in this definition, Eqs. (37), (42), (43), and (45) have been used), the amplitudes

$$
A_{\eta}=\max _{1 \leqslant n \leqslant N} \eta_{n}, \quad A_{\varphi}=\max _{1 \leqslant n \leqslant N} \varphi_{n}, \quad A_{\rho}=\min _{1 \leqslant n \leqslant N} \eta_{n},
$$

and the mean-root-square width

$$
L=2\left(\sum_{n=1}^{N}\left(n-n_{c}\right)^{2} \rho_{n} / R\right)^{1 / 2}
$$

where

$$
R=\sum_{n=1}^{N} \rho_{n}
$$

is the total compression of the helix backbone and
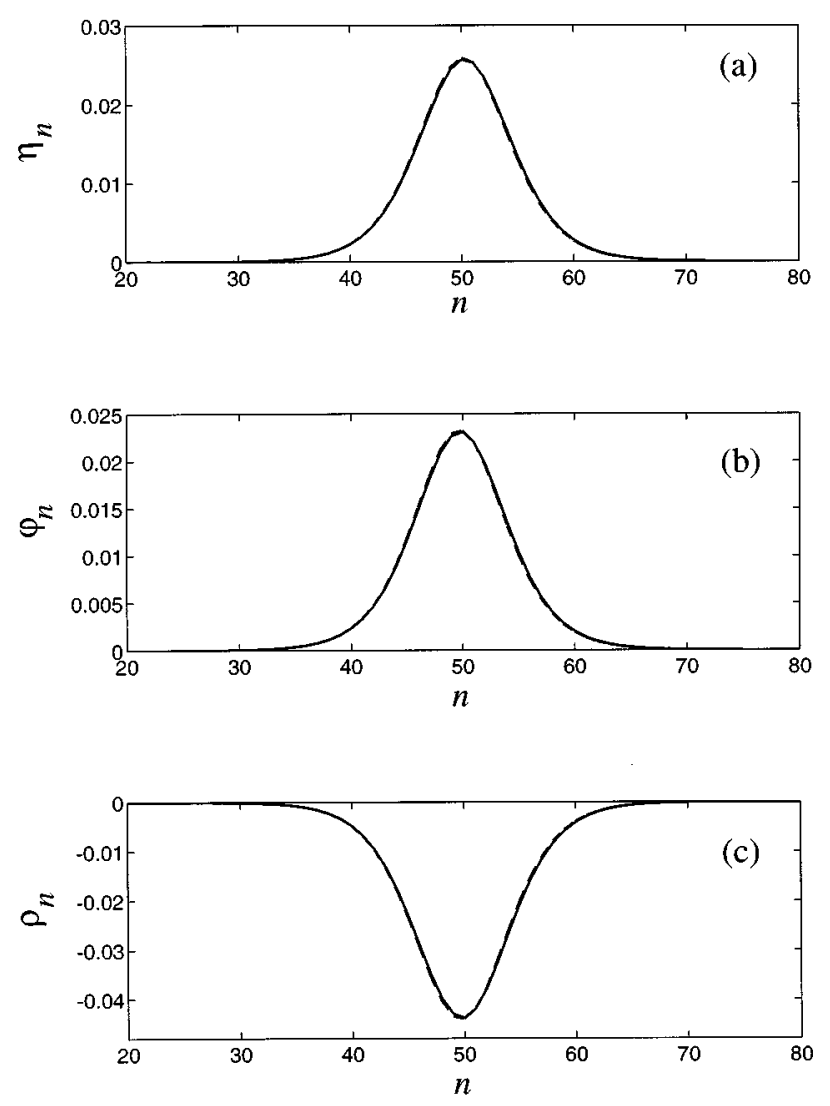

FIG. 4. The three-component profile of the soliton in the helix backbone with the parameter values (35) and the anharmonicities $\gamma_{1}=\gamma_{2}=0$ and $\gamma_{3}=1$ at the initial instant of time $\tau=0$ (dashed line) and at the final instant $\tau=28062.45$ when the soliton has passed 100000 chain sites (solid line). The initial velocity of the soliton was $s=1.05 s_{l}$.

$$
n_{c}=\frac{1}{2}+\sum_{n=1}^{N} n \rho_{n} / R
$$

is the position of the soliton center.

\section{NUMERICAL RESULTS}

In this section we will numerically find three-component soliton solutions, and study their stability properties for the $\alpha$-helix backbone with the parameter values (35). The nonlinearity of the dynamics of the $\alpha$-helix protein molecules in the first turn is caused by the anharmonicity of soft hydrogen bonds. Therefore we will take into account only the anharmonicity of the third neighbors $(\nu=3)$, i.e., we take $\gamma_{1}$ $=\gamma_{2}=0$ and $\gamma_{3}>0$. Let us find soliton solutions for three values of the anharmonicity: $\gamma_{3}=0.1,1$, and 10 . The numerical solution of systems (47), (48), and (50) has shown that at weak anharmonicity (e.g., $\gamma_{3}=0.1$ ), there are no soliton solutions, while for the value $\gamma_{3}=1$ the soliton solutions exist only with velocities in the segment $1<s / s_{l}<1.065$. In this interval of velocities, the solutions have bell-shaped soliton profiles, smoothly depending on the chain site, as illustrated by Fig. 4. The dependences of the energy $E$, width $L$, and amplitudes $A_{\eta}, A_{\varphi}$, and $A_{\rho}$ of the soliton on its dimensionless velocity $s / s_{l}$ are given in Table I. As follows 
TABLE I. Dependence of the energy $E$, width $L$, and amplitudes $A_{\eta}, A_{\varphi}$, and $A_{\rho}$ of the soliton on its velocity $s / s_{l}$ for $\gamma_{3}=1$.

\begin{tabular}{lccccc}
\hline \hline$s / s_{l}$ & $E$ & $L$ & $A_{\eta}$ & $A_{\varphi}$ & $A_{\rho}$ \\
\hline 1.01 & 0.02026 & 20.79 & 0.00539 & 0.00488 & -0.00929 \\
1.02 & 0.05763 & 15.19 & 0.01062 & 0.00963 & -0.01830 \\
1.03 & 0.10669 & 11.01 & 0.01574 & 0.01429 & -0.02711 \\
1.04 & 0.16557 & 10.01 & 0.02082 & 0.01878 & -0.03574 \\
1.05 & 0.23326 & 9.61 & 0.02576 & 0.02327 & -0.04417 \\
1.06 & 0.30910 & 9.50 & 0.03055 & 0.02761 & -0.05221 \\
\hline \hline
\end{tabular}

from Fig. 4, in the region of the localization of the soliton solution, compression of the chain occurs, accompanied by localized thickening and untwisting. The solitons can exist only in a narrow interval of supersonic velocities. When $s \rightarrow s_{l}$, the soliton energy and amplitudes monotonically tend to zero, while the width increases to infinity. With the growth of the velocity, the energy and absolute values of the amplitudes monotonically increase, whereas the width monotonically decreases. For strong anharmonicity, the soliton has a finite supersonic speed spectrum. Thus at $\gamma_{3}=10$ we have the segment $1<s / s_{l}<1.065$. For this case, the dependences of the energy $E$, width $L$, and amplitudes $A_{\eta}, A_{\varphi}$, and $A_{\rho}$ on the velocity $s / s_{l}$ are illustrated by Table II.

The second stage of our numerical studies was the simulation of the dynamics of the soliton profiles obtained above by solving the nonlinear algebraic equations (47), (48), and (50). To this end, we considered the dynamics in a finite chain with fixed ends. The dynamics of such a system is governed by the system of the equations of motion (39)-(41) with $4 \leqslant n \leqslant N-3$, where $N$ is the number of molecules in the chain. The displacements $\eta_{n}, \theta_{n}$, and $\beta_{n}$ of the molecules at the chain ends, with the subscripts $n=1,2,3$, $N-2, N-1$, and $N$ are assumed to be fixed. Let $\left\{\eta_{n}^{0}, \varphi_{n}^{0}, \rho_{n}^{0}\right\}_{n=1}^{N}$ be a soliton solution obtained by solving the system of Eqs. (47), (48), and (50). Then the soliton center is found at the $(N / 2)$ th chain site, and this solution is used to construct the initial conditions for numerical integration of Eqs. (39)-(41) as follows. First, we note that in order to simulate the propagation of the soliton in an infinite chain, instead of the chain consisting of $N$ sites, we choose the initial conditions in the "prolonged" chain, which contains $2 N$ sites, according to the relations

$$
\begin{gathered}
\eta_{n}(0)=\eta_{n}^{0}, \quad n=1, \ldots, N, \quad \eta_{n}(0)=\eta_{N}^{0}, \quad n=N+1, \ldots, 2 N \\
\theta_{1}(0)=0, \quad \theta_{n+1}(0)=\theta_{n}(0)+\varphi_{n}^{0}, \quad n=1, \ldots, N, \\
\theta_{n}(0)=\theta_{N+1}(0), \quad n=N+2, \ldots, 2 N
\end{gathered}
$$

$$
\begin{gathered}
\beta_{1}(0), \quad \beta_{n+1}(0)=\beta_{n}(0)+\rho_{n}^{0}, \quad n=1, \ldots, N, \\
\beta_{n}(0)=\beta_{N+1}(0), \quad n=N+2, \ldots, 2 N, \\
\eta_{n}^{\prime}(0)=-s\left[\eta_{n+1}(0)-\eta_{n-1}(0)\right] / 2 h, \\
\theta_{n}^{\prime}(0)=-s\left[\theta_{n+1}(0)-\theta_{n-1}(0)\right] / 2 h, \\
\beta_{n}^{\prime}(0)=-s\left[\beta_{n+1}(0)-\beta_{n-1}(0)\right] / 2 h, \quad n=4, \ldots, 2 N-3, \\
\eta_{j}^{\prime}=\theta_{j}^{\prime}=\beta_{j}^{\prime}=\eta_{2 N-j}^{\prime}=\theta_{2 N-j}^{\prime}=\beta_{2 N-j}^{\prime}=0, \quad j=1,2,3
\end{gathered}
$$

where the prime denotes the differentiation with respect to the dimensionless time $\tau$. Next we shift the soliton profile back by $N$ sites just when it passes $N$ sites, i.e., when $\beta_{3 N / 2}(\tau)$ becomes greater than $\beta_{N / 2}(0)$; we accomplish the substitutions

$$
\begin{gathered}
\eta_{N+n}(\tau)=\eta_{n}(\tau), \quad \theta_{N+n}(\tau)=\theta_{n}(\tau), \quad \beta_{N+n}(\tau)=\beta_{n}(\tau), \\
\eta_{N+n}^{\prime}(\tau)=\eta_{n}^{\prime}(\tau), \quad \theta_{N+n}^{\prime}(\tau)=\theta_{n}^{\prime}(\tau), \\
\beta_{N+n}^{\prime}(\tau)=\beta_{n}^{\prime}(\tau), \quad n=1, \ldots, N \\
\eta_{n}(\tau)=\eta_{2 N}(0), \quad \theta_{n}(\tau)=\theta_{2 N}(0), \quad \beta_{n}(\tau)=\beta_{2 N}(0), \\
\eta_{n}^{\prime}(\tau)=0, \quad \theta_{n}^{\prime}(\tau)=0, \quad \beta_{n}^{\prime}(\tau)=0, \quad n=N+1, \ldots, 2 N
\end{gathered}
$$

After each such shift, we compare the current soliton profile with its initial shape. To this end, we define the "distance", function

$$
d(\tau)=1-\frac{1}{3}\left[\sqrt{S_{\eta}(\tau) / R_{\eta}}+\sqrt{S_{\varphi}(\tau) / R_{\varphi}}+\sqrt{S_{\rho}(\tau) / R_{\rho}}\right]
$$

where

TABLE II. Dependence of the energy $E$, width $L$, and amplitudes $A_{\eta}, A_{\varphi}$, and $A_{\rho}$ on the velocity $s / s_{l}$ for $\gamma_{3}=10$.

\begin{tabular}{cccccc}
\hline \hline$s / s_{l}$ & $E$ & $L$ & $A_{\eta}$ & $A_{\varphi}$ & $A_{\rho}$ \\
\hline 1.01 & 0.00022 & 20.82 & 0.00057 & 0.00051 & -0.00097 \\
1.02 & 0.00064 & 15.23 & 0.00114 & 0.00100 & -0.00192 \\
1.03 & 0.00118 & 12.61 & 0.00170 & 0.00148 & -0.00284 \\
1.04 & 0.00183 & 11.05 & 0.00226 & 0.00195 & -0.00374 \\
1.05 & 0.00257 & 10.06 & 0.00281 & 0.00241 & -0.00464 \\
1.06 & 0.00341 & 9.58 & 0.00336 & 0.00286 & -0.00551 \\
\hline \hline
\end{tabular}




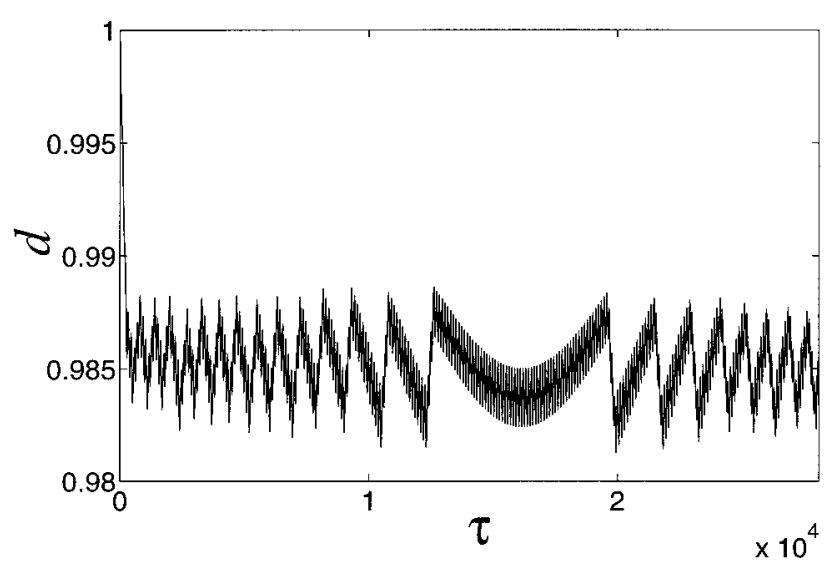

FIG. 5. Dependence of the function $d$, describing the change of the soliton shape, on time $\tau$ for the soliton with the initial velocity $s=1.05 s_{l}$ in the helix chain with the same parameter values as in Fig. 4.

$$
\begin{gathered}
S_{\eta}(\tau)=\sum_{n=1}^{2 N}\left[\eta_{n}(\tau)-\eta_{n}(0)\right]^{2}, \\
S_{\varphi}(\tau)=\sum_{n=1}^{2 N-1}\left[\varphi_{n}(\tau)-\varphi_{n}(0)\right]^{2}, \\
S_{\rho}(\tau)=\sum_{n=1}^{2 N-1}\left[\rho_{n}(\tau)-\rho_{n}(0)\right]^{2}, \\
R_{\eta}=\sum_{n=1}^{2 N} \eta_{n}^{2}(0), \quad R_{\varphi}=\sum_{n=1}^{2 N-1} \varphi_{n}^{2}(0), \quad R_{\rho}=\sum_{n=1}^{2 N-1} \rho_{n}^{2}(0) .
\end{gathered}
$$

Function (61) allows us to estimate the change of the soliton shape. When the current and initial profiles coincide perfectly, then $d=1$. Otherwise, $d<1$.

We have studied the soliton dynamics in the chain consisting of $N=100$ molecules with the parameters (35) when $\gamma_{1}=\gamma_{2}=0$ and $\gamma_{3}=1$. When the initial speed was $s / s_{l}$ $=1.05(s=3.5645)$, then the soliton passed 100000 chain sites during the time $\tau=28062.45$, and this propagation corresponds to the velocity $s=100000 / \tau=3.5635=1.0497 s_{l}$. As can be seen from Fig. 4, the soliton shape at the final instant of time practically coincides with the initial profile. The change of the soliton shape in time, described by the function $d=d(\tau)$ [see Eqs. (61) and (62)], is given in Fig. 5. Its behavior demonstrates small oscillations around the mean value $\bar{d}=0.985$. The nonsmooth behavior of the distance $d(\tau)$ appears due to incommensurateness of the velocity of motion with the value of the discrete step of integration $\Delta \tau$. The system of the equations of motion (39)-(41) was integrated by the fourth-order Runge-Kutta method with the constant step of integration $\Delta \tau=0.02$. The discreteness of time does not allow us exactly to find an instant of time when the soliton has passed exactly 200 chain sites. Therefore some small shifts of the soliton center occur and, as a result, small-amplitude oscillations appear in the function $d(\tau)$. Simulations of the equations of motion have shown
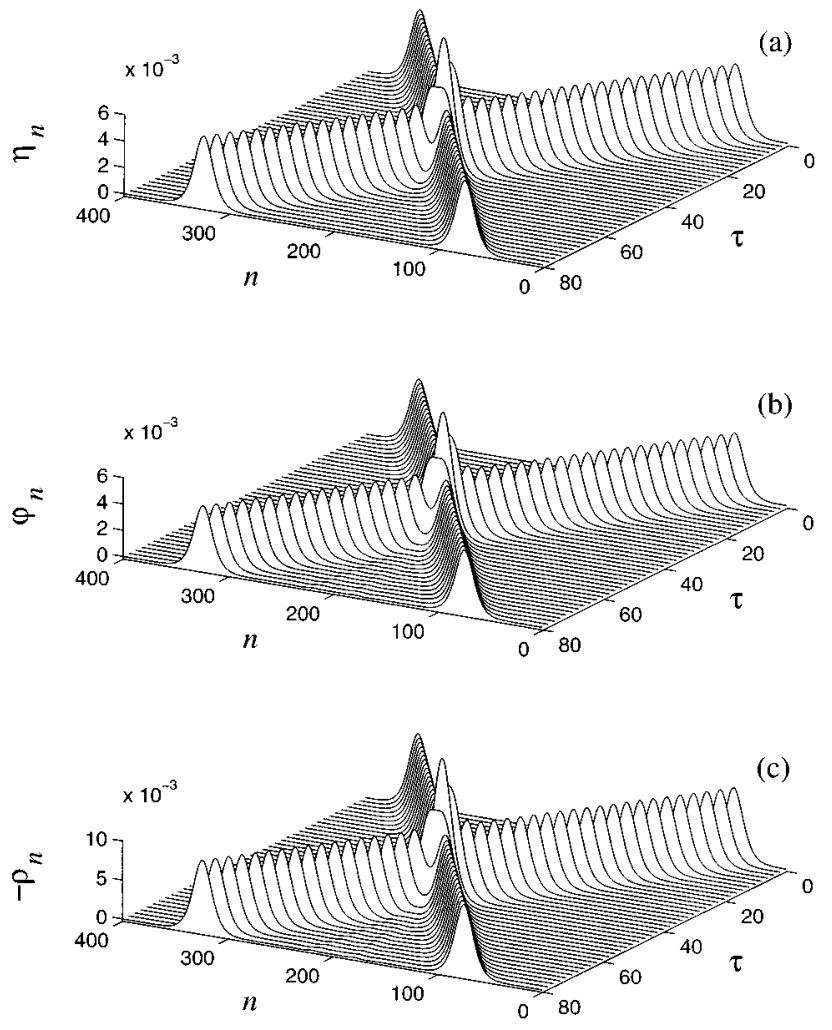

FIG. 6. Elastic collision of the solitons in the chain backbone with the same parameter values as in Fig. 4. Both the solitons propagate with the velocity $s=1.01 s_{l}=3.4287$.

that the numerical procedure of looking for soliton solutions gives correct soliton profiles, and solitons themselves are dynamically stable.

Consider now the head-in collision of the solitons. The simulations have shown that for velocities close to the speed of longitudinal sound, the collision of solitons occurs practically elastically without emission of small-amplitude waves (see Fig. 6). However, for higher velocities, close to the upper edge of the segment of admissible velocities ("velocity spectrum"), the interaction of solitons becomes inelastic. The collision leads to the emission of small-amplitude waves as illustrated by Fig. 7.

In finite $\alpha$-helix chains, the solitons can be created at the ends of the chain. Therefore, we have considered the dynamics of the chain when it is initially compressed at one of the ends. To this end, for the simulations of Eqs. (39)-(41), we have chosen the following initial conditions:

$$
\begin{gathered}
\eta_{n}(0)=0, \quad \eta_{n}^{\prime}(0)=0, \quad \theta_{n}(0)=0, \quad \theta_{n}^{\prime}(0)=0, \\
\beta_{n}^{\prime}(0)=0, \quad n=1, \ldots, N ; \\
\beta_{1}(0)=\beta_{2}(0)=\beta_{3}(0)=A, \quad \beta_{n}(0)=0, \quad n=4, \ldots, N .
\end{gathered}
$$

Here $A>0$ is the amplitude of the initial compression at the first three chain sites of the left end, and $N=2000$ is the total number of sites chosen for the simulations. Again, we take $\gamma_{1}=\gamma_{2}=0$ and $\gamma_{3}>0$. The simulations have shown that, for the weak anharmonicity $\gamma_{3}=0.1$, the initial compression of the chain leads only to the appearance of an oscillating wave packet propagating with subsonic velocities. Supersonic soli- 

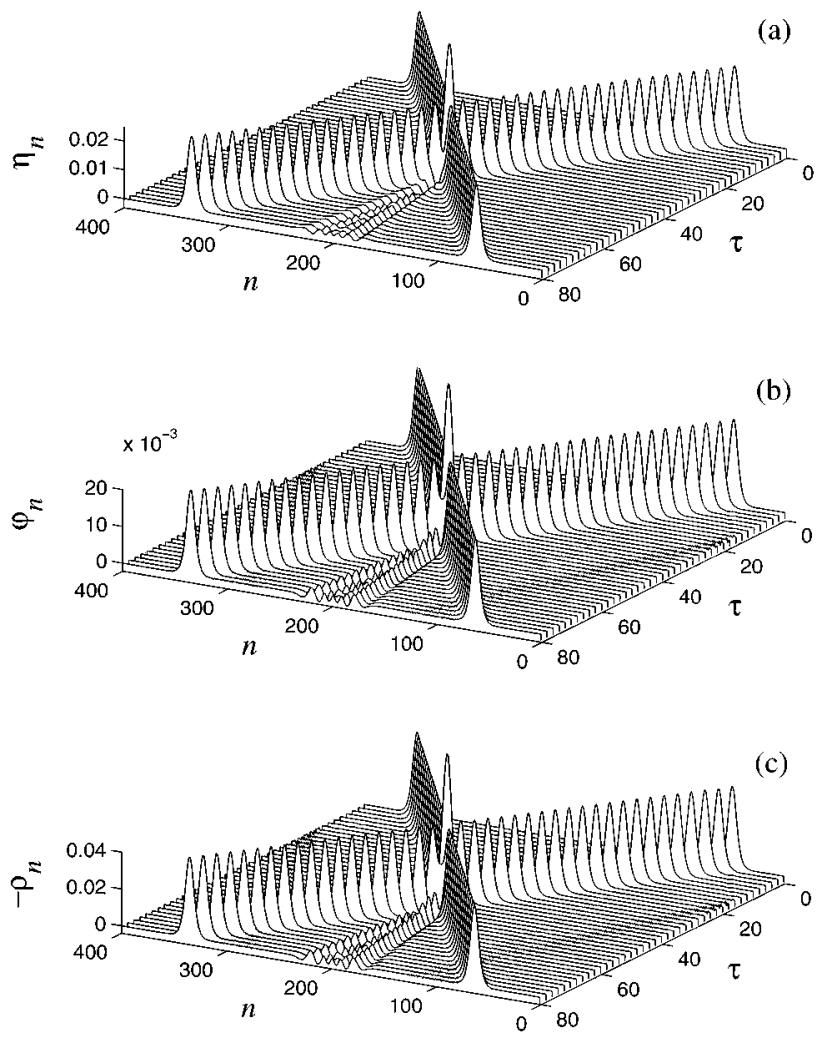

FIG. 7. Inelastic collision of the solitons in the backbone with the same parameter values as in Fig. 4. The initial velocity of the solitons is $s=1.05 s_{l}=3.5645$.

tons are not formed, and their absence is in accordance with the result obtained above for the backbone with weak anharmonicity. In the case with middle anharmonicity, the compression with the amplitudes $A=0.1$ and 0.2 also resulted in the appearance of a subsonic wave packet. However, the compression with larger amplitudes also resulted in the appearance of a supersonic soliton. Thus, for $A=0.3$, the soliton had the velocity $s / s_{l}=1.031$ and for $A=0.4$, the velocity was $s=1.049$. For the stronger anharmonicity, $\gamma_{3}=10$, the compression with the amplitude $A=0.05$ resulted in the formation of a supersonic soliton with the velocity $s / s_{l}$ $=1.066$ (see Fig. 8). The presence of the two sound velocities $s_{t}$ and $s_{l}$ leads to the appearance of two oscillating wave packets (torsional and longitudinal waves, respectively) which are clearly seen in Fig. 8. The compression with the larger amplitude $A=0.1$ leads to the formation of the soliton like supersonic pulse with the velocity $s / s_{l}=1.175$ (see Fig. 9). This value of the velocity is outside the soliton velocity spectrum. Therefore the motion of such a soliton is accompanied by the continuous emission of small-amplitude waves (phonons). In an infinite chain, such emission should result in decreasing the soliton velocity, approaching the upper edge of the soliton velocity spectrum; however, in a finite chain, the soliton has no time to throw down its velocity. Thus these simulations of the time evolution of a narrow initial pulse have confirmed our conclusions drawn above on the finiteness of the supersonic soliton velocity spectrum on one side, and the absence of soliton solutions for weak anharmonicity on the other side.

Finally, we note that the accuracy of the numerical inte-

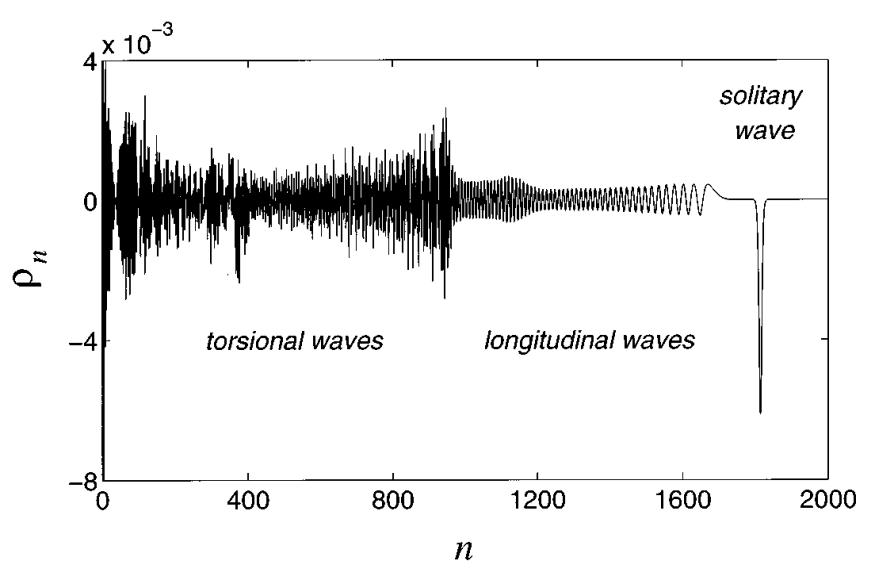

FIG. 8. Formation of the supersonic soliton and of two wave packets in the helix backbone under the initial $(\tau=0)$ compression of the three bonds at its left end. The parameters of the backbone are given by Eqs. (35), $\gamma_{1}=\gamma_{2}=0$, and $\gamma_{3}=10$. The amplitude of the initial compression is $A=0.05$. The distribution of the longitudinal relative displacements $\rho_{n}$ 's along the helix chain is plotted at the instant $\tau=500$.

gration of Eqs. (39)-(41) was estimated through the conservation of the integral of the total energy [see Eq. (37)]

$$
\begin{aligned}
\mathcal{H}= & \sum_{n=4}^{N-3}\left\{\frac{1}{2}\left[\left(\frac{d \eta_{n}}{d \tau}\right)^{2}+\left(1+\eta_{n}\right)^{2}\left(\frac{d \theta_{n}}{d \tau}\right)^{2}+\left(\frac{d \beta_{n}}{d \tau}\right)^{2}\right]\right. \\
& \left.+\sum_{j} U_{j}\left(r_{j n}\right)\right\} .
\end{aligned}
$$

The value of the integration step $\Delta \tau=0.02$, used in the present paper, ensured the conservation of integral (64) with an accuracy up to six digits during the whole time of the numerical integration.

\section{CONCLUSION AND DISCUSSIONS}

It was an attractive point of view to study the transport of vibrational energy in biopolymers such as protein on the ba-

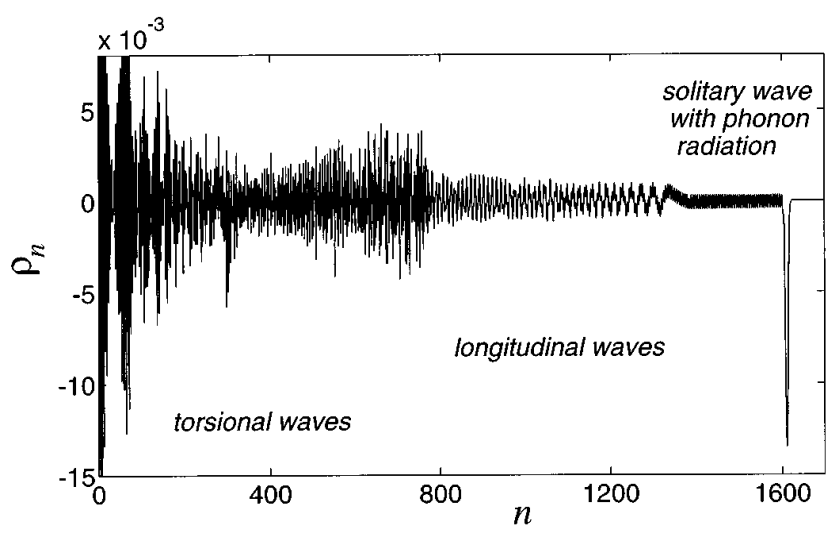

FIG. 9. Formation of the supersonic soliton emitting smallamplitude waves and of two wave packets in the helix backbone with the same parameter values as in Fig. 8 under the initial $(\tau$ $=0$ ) compression of the left end bonds. The amplitude of the initial compression is $A=0.1$. The distribution of the longitudinal relative displacements $\rho_{n}$ 's along the chain is given at $\tau=400$. 
sis of $1 \mathrm{D}$ nonlinear lattice models. The first attempt in this direction was formulated by Davydov with co-workers (see the Refs. [5] and [6], and references therein) who suggested that the intramolecular vibrational amide- $I$ mode could be self-trapped through its interaction with deformation in the protein structure. Further, Davydov and co-workers [28,29] and Scott [30] generalized this theory by taking dipoledipole coupling between the three spines into account. While the Davydov model and the related models (the Takeno model [31] should also be mentioned) considered intramolecular modes, Yomosa [8] modeled the energy transfer in protein, essentially applying the Fermi-Pasta-Ulam model. The present paper also focuses only on the large-amplitude supersonic lattice solitons, so that we do not consider any intramolecular modes. All intermolecular (or interatomic) interactions in our model are represented by pair central forces. However, in order somehow to stabilize a chain backbone and to fix a single ground state, except for the nearestneighboring interactions, we should take into account some additional intermolecular forces, including next or more remote neighbors. As a result, the latter forces in biology form secondary structure, i.e., some regular spatial configuration of the chain. In the simplest case, such a configuration has naturally the form of a so-called $3_{10}$ helix. Therefore Olsen, Lomdahl, and Kerr [10] considered two types of intermolecular forces: between the first neighbors and between the nearest neighbors along the longitudinal direction. In order to have a completely stabilized helix backbone in the 3D space, we incorporated in the helix model a third type of intermolecular forces, namely, between the second neighbors. For instance, in the case of the $3_{10}$-helix backbone, our model contains the first-, second-, and third-neighboring intermolecular forces. Alternatively, instead of this coupling, a threebody force, which fixes the valence angle $\psi$, could be considered. Any of these force constraints is sufficient for a helix backbone to have a single ground state. In this paper we restricted ourselves to the case when the chain molecules are coupled only through central forces of spherical symmetry. Given in terms of valence bonds, the helix Hamiltonian becomes more complicated. Therefore the Hamiltonian (15) may be considered as the most simple 3D generalization of the 1D FPU chain.

Thus, in the present paper the standard 1D FPU model has been generalized to the three spatial dimensions when each chain molecule is allowed to have three degrees of freedom. The resulting geometric structure is a helix backbone with $\nu \geqslant 3$ spines. Three-component nontopological solitons have been shown to exist and propagate with supersonic velocities in the helix backbone with any number of spines. Similarly to the 1D case, for the existence of the soliton solutions, the presence of anharmonicity, at least, in the longitudinal (hydrogen) bonds connecting each $n$th and $(n+\nu)$ th molecules, is a necessary condition. However, compared to the 1D FPU model, the existence of stable soliton solutions in the 3D helix backbone appears to be more limited. First, they exist if the anharmonicity of the $\nu$-neighboring forces (longitudinal bonds) is sufficiently strong. Second, the segment of admissible (supersonic) velocities of solitons is always finite. It is quite narrow and for all velocities from this segment, and the soliton propagation is uniform, retaining velocity and profile. However, when the solitons collide, their behavior after col- lision depends on velocity. At the lower edge of the segment (soliton velocity spectrum), the soliton collision is elastic, whereas, for higher velocities, at the upper edge the solitons collide inelastically with the radiation of small-amplitude waves. Summarizing, we conclude that in a 3D isolated helix backbone the three-component solitons, describing supersonic pulses of longitudinal backbone compression, still exist as dynamically stable objects. However, the range of their existence is more limited if compared to the 1D FPU lattice.

Since the present paper focuses mainly on the problem of how to find pure soliton solutions, in order to check the stability of the soliton solutions obtained under the minimization procedure, as well as to demonstrate the accuracy of the method for seeking these solutions, we performed numerical simulations of waves traveling over 100000 chain sites. Of course, it is very rare for realistic biological macromolecules to be uniform over such long lengths. Thus a protein which consists of amino acids should be modeled by a chain with mass variation. We performed numerical simulations of the time evolution of the lattice solitons in the 1D FPU chain with randomly distributed masses, and found that their propagation through disordered segment is, in general, unstable. Thus, if the mass impurity is sufficiently small, then emission of small-amplitude waves occurs when the soliton passes through the impurity. For larger impurities, splitting the soliton, for instance, into two solitons, one of these reflects the impurity. If the impurity is very large, then inelastic reflection of the soliton takes place. Assuming a soliton to be incident on a disordered segment of the chain embedded between two homogeneous semi-infinite chains, the transmission coefficient of the soliton energy could be calculated. In the 1D case, this problem was solved numerically in Ref. [32].

We do not expect that the 1D results will change crucially for the helix chain. At least, they should be similar at the lower edge of the soliton velocity spectrum where the soliton profile is broad. However, in any case we have energy loss and eventually soliton breaking. The scattering of the lattice solitons on mass impurities is due to their high (supersonic) velocities. Slow solitons such as envelope solitons or topological defects are much more stable with respect to impurities. Therefore, from the point of view of "soliton transparency" in real biological macromolecules, the Davydov-Scott self-trapping mechanism seems to be much more promising, as Davydov's soliton does not loose energy at all, at least for sufficiently small velocities [33]. However, it should be noted that the helix part of the Davydov-Scott "excitonhelix" model $[28-30,34,35]$ is simplified because only longitudinal displacements of the chain molecules are considered. Though transverse displacements along the radial directions of the helix backbone can be taken into account [29], nevertheless the model is still one-dimensional because the intermolecular interactions are considered only in the longitudinal direction. The coupling between the spines is incorporated only through a semiclassical term, namely, the transverse dipole-dipole interaction energy between the nearest-neighbor amino acids. It is important to note that on the basis of this oversimplified exciton-helix model, the numerical calculations by Scott and co-workers $[30,34,35]$ discovered the threshold of the exciton-phonon coupling for formation of the soliton states in protein, while, in the cor- 
responding single chain, the self-trapping occurs at any value of this coupling. Therefore it is not obvious what happens with the intramolecular mode when the lattice (helix) subsystem is completed properly, as described in this paper. From this point of view, the present work may be considered as a first step to study more carefully the Davydov-Scott exciton-helix model. Therefore the numerical simulations performed by Scott and co-workers [30,34,35] should be extended to the case when the helix part of the Hamiltonian is given by Eq. (10). Moreover, since the helix subsystem changes significantly (three degrees of freedom for the chain molecule motions and three types of intermolecular interactions are involved), the corresponding Langevin equations will essentially differ from those used by Lomdahl and Kerr [36]. Therefore their results on the thermal stability of Davydov's soliton should be revised in the "complete" excitonhelix model. Thus, semiclassical investigations in the spirit of Refs. [30, 34, 35], on one hand, and numerical simulations similar to those of Lomdahl and Kerr [36] and Lawrence et al. [37], on the other hand, are of great importance in the future.

\section{ACKNOWLEDGMENTS}

This work was partially carried out with the financial support from the European Economic Community (EEC) under the INTAS, Grant No. 94-3754. Both of us (A.V.S. and A.V.Z.) would also like to express our gratitude to the MIDIT Center and the Department of Mathematical Modelling of The Technical University of Denmark for financial support and hospitality. Stimulating and useful discussions with A. C. Scott, as well as his suggestions about the process of this investigation, are gratefully acknowledged.
[1] E. Fermi, J. Pasta, and S. Ulam, Collected Works of Enrico Fermi (University of Chicago, Chicago, 1965), Vol. II, p. 978.

[2] N. J. Zabusky and M. D. Kruskal, Phys. Rev. Lett. 15, 241 (1965).

[3] N. J. Zabusky, Comput. Phys. Commun. 5, 1 (1973).

[4] M. Toda, Phys. Rep. 18, 1 (1975).

[5] A. S. Davydov, Solitons in Molecular Systems (Reidel, Dordrecht, 1985).

[6] A. C. Scott, Phys. Rep. 217, 1 (1992).

[7] M. A. Collins, Chem. Phys. Lett. 77, 342 (1981).

[8] S. Yomosa, Phys. Rev. A 32, 1752 (1985).

[9] O. H. Olsen, M. R. Samuelsen, S. B. Petersen, and L. Nørskov, Phys. Rev. A 38, 5856 (1988); 39, 3130 (1989).

[10] O. H. Olsen, P. S. Lomdahl, and W. C. Kerr, Phys. Lett. A 136, 402 (1989).

[11] P. S. Lomdahl, O. H. Olsen, and M. R. Samuelsen, Phys. Lett. A 152, 343 (1991).

[12] P. L. Christiansen, P. S. Lomdahl, and V. Muto, Nonlinearity 4, 477 (1990).

[13] V. Muto, P. S. Lomdahl, and P. L. Christiansen, Phys. Rev. A 42, 7452 (1990).

[14] J. Pouget, S. Aubry, A. R. Bishop, and P. S. Lomdahl, Phys. Rev. B 39, 9500 (1989).

[15] O. M. Braun and Yu. S. Kivshar, Phys. Rev. B 44, 7694 (1991).

[16] O. M. Braun, O. A. Chubykalo, Yu. S. Kivshar, and L. Vázquez, Phys. Rev. B 48, 3734 (1993).

[17] S. K. Turitsyn, Phys. Rev. E 47, R796 (1993).

[18] M. Peyrard and A. R. Bishop, Phys. Rev. Lett. 62, 2755 (1989).

[19] M. Peyrard, T. Dauxois, H. Hoyet, and C. R. Willis, Physica D 68, 104 (1993).
[20] T. Dauxois, M. Peyrard, and A. R. Bishop, Phys. Rev. E 47, R44 (1993).

[21] A. V. Zolotaryuk, P. L. Christiansen, and A. V. Savin, Phys. Rev. E 54, 3881 (1996).

[22] S. Cadet, Phys. Rev. Lett. 121, 77 (1987).

[23] N. Flytzanis, St. Pnevmatikos, and M. Remoissenet, J. Phys. C 18, 4603 (1985).

[24] N. Flytzanis, St. Pnevmatikos, and M. Peyrard, J. Phys. A 22, 783 (1989).

[25] J. C. Eilbeck and R. Flesch, Phys. Lett. A 149, 200 (1990).

[26] D. B. Duncan, J. C. Eilbeck, H. Feddersen, and J. A. D. Wattis, Physica D 68, 1 (1993).

[27] D. F. Shanno and K. H. Phua, ACM Trans. Math. Softw. 6, 618 (1980).

[28] A. S. Davydov, A. A. Eremko, and A. I. Sergienko, Ukr. Fiz. Zh. 23, 983 (1978).

[29] A. A. Eremko and A. I. Sergienko, Ukr. Fiz. Zh. 25, 2013 (1980).

[30] A. C. Scott, Phys. Rev. A 26, 578 (1982).

[31] S. Takeno, Prog. Theor. Phys. 71, 395 (1984); 73, 853 (1985).

[32] Q. Li, St. Pnevmatikos, E. N. Economou, and C. M. Soukoulis, Phys. Rev. B 37, 3534 (1988); Q. Li, C. M. Soukoulis, St. Pnevmatikos, and E. N. Economou, ibid. 38, 11888 (1988).

[33] A. V. Zolotaryuk, K. H. Spatschek, and O. Kluth, Phys. Rev. B 47, 7827 (1993).

[34] A. C. Scott, Phys. Scr. 29, 279 (1984); L. MacNeil and A. C. Scott, ibid. 29, 284 (1984).

[35] J. M. Hyman, D. W. McLaughlin, and A. C. Scott, Physica D 3, 23 (1981).

[36] P. S. Lomdahl and W. C. Kerr, Phys. Rev. Lett. 55, 1235 (1985).

[37] A. F. Lawrence, J. C. McDaniel, D. B. Chang, B. M. Pierce, and R. R. Birge, Phys. Rev. A 33, 1188 (1986). 